\title{
SPATIAL DISTRIBUTION OF FELT INTENSITIES FOR PORTUGAL EARTHQUAKES
}

\author{
C. Catita ${ }^{1,2}$, M. P. Teves-Costa ${ }^{1,2}$, L. Matias ${ }^{1,2}$, J. Batlló $^{3}$ \\ ${ }^{1}$ IDL, Instituto Dom Luiz, Lisbon, Portugal- (cmcatita, mpcosta, lmmatias)@fc.ul.pt \\ ${ }^{2}$ Faculty of Sciences of University of Lisbon, Portugal \\ ${ }^{3}$ Institut Cartogràfic i Geològic de Catalunya, Barcelona, Spain- Josep.Batllo@icgc.cat
}

KEY WORDS: Macroseismic intensities; GIS; spatial autocorrelation; geostatistics; spatial pattern; Portugal

\begin{abstract}
:
Macroseismic intensity is a parameter of the severity of ground movement evaluated in terms of the effects that earthquakes produce on people, buildings and the environment, constituting an extremely important information source for the seismologist and engineer of structures. In recent studies, the authors of this study published a new map of maximum intensities observed for continental Portugal considering all observations of macro-seismic intensity higher than III relative to earthquakes occurred between 1344 and 2015 (Teves-Costa et al., 2019). In each parish and municipality of the continental part of the country, the maximum values of intensity were defined. A map of maximum intensities produced in this way was essentially controlled by earthquakes of greater intensity, the 1909.04.23 (near-field) and 1755.11.01 (far-field) earthquakes. In the present work, a spatial distribution of the earthquakes presents in the database (classified by their nature - near-field or far-field - intensity, and epoch) are analysed. The spatial structure, which allows the detection of spatial dependence or autocorrelation of intensity values is also explored in this work, in order to identify regions with similar macroseismic characteristics. The combination of the map of maximum intensities and the products generated in this work are essential for the authorities as a base of support in the definition of joint adaptation strategies for the various regions of the territory, as well as contribute to a better management of the emergency system in Portugal.
\end{abstract}

\section{INTRODUCTION}

\subsection{Geodynamic framework}

The geodynamic framework of Portugal mainland is dominated by the collision between the Nubian and Eurasian plates which occurs at a very slow speed of $\sim 4 \mathrm{~mm} /$ year. The consequent tectonic activity, that also extends in an emerging region, leading to a slow deformation regime in the continental Portuguese territory. The seismic cycles associated with active faults are therefore very long and seismic hazard assessment studies should consider the historical seismicity of Portugal mainland as an undoubted source of information. Despite the existence of moderate seismic activity, due to this geotectonic context, earthquakes of high magnitude occur as well, such was the $1^{\text {st }}$ November 1755 Lisbon earthquake with an estimated magnitude of $\mathrm{Mw}$ 8.5-8.7. In Europe this event caused considerable damage in Spain - particularly in Madrid and Seville. Shaking was felt in France, Switzerland, and Northern Italy too, and the following tsunami also caused many victims and destruction on some coastal areas of Portugal. The last strong earthquake that affected the territory of Portugal mainland occurred on February 28, $1969(\mathrm{M} \approx 8.0)$. Its epicentre is located in the sea, on the SW of Cape S. Vicente, in the Abissal Plain of Ferradura and, according to several authors, in the same seismogenic zone where the earthquake of November 1, $1755(\mathrm{M} \geq 8.2)$. The earthquake of 1969.02.28 was felt throughout the continental territory with intensities between IV and VIII (MM56). In the SW region of Portugal, it produced the collapse of some houses and in Lisbon (intensity V-VI) some chimneys fell.

Prior seismic hazard studies performed for the elaboration of the Portuguese EC8 (IPQ 2010) grouped the potential seismogenic structures in 11 zones (Figure 1).

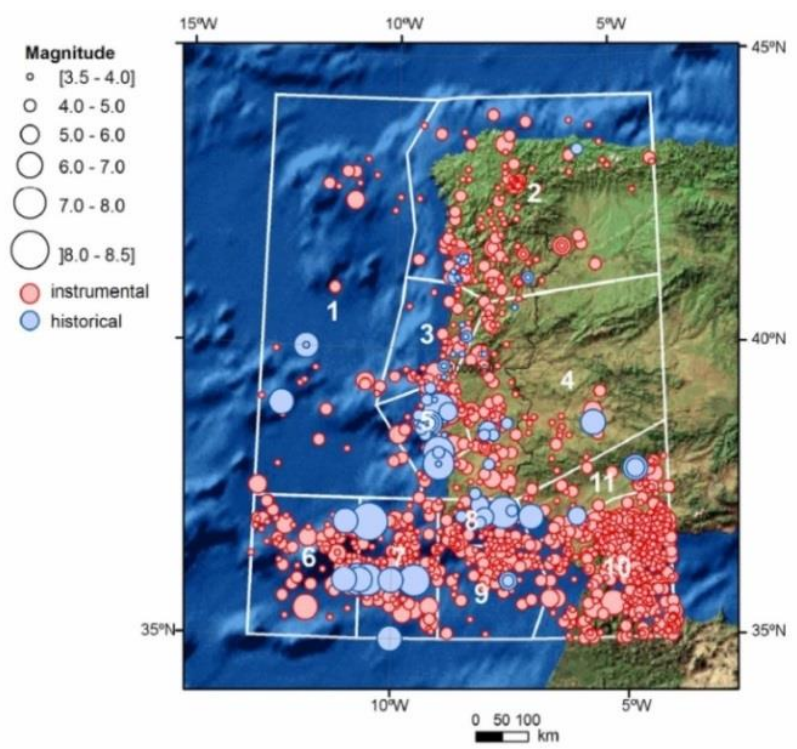

Figure 1. Seismic zonation map used to support EC8 code zoning for Portugal (IPQ, 2010) (adapted from Campos Costa et al. 2008)

\subsection{Seismic intensities database}

One essential tool for seismic risk management is mapping the maximum intensities. This map should represent at each site the maximum macroseismic intensity that has been observed since historical times. In the absence of observations, it should provide for each site an estimate of that maximum intensity as a consequence of known earthquakes. The current map of maximum intensities for mainland Portugal was elaborated in the 80 's of last century by the National Institute of Meteorology and Geophysics (current Portuguese Institute of the Sea and the Atmosphere, IPMA), based on the isoseismal map of the main 
earthquakes felt (e.g. earthquakes of 1531, 1755, 1909 and 1969), to which was added isoseismal information of some earthquakes in Spain. The resulting maximum intensities map considers the intensities observed in the main earthquakes of the past, but does not consider, for example, the occurrence of earthquakes of smaller magnitude with a significant local effect and that may have produced, for some places, intensities higher than those observed in the great earthquakes. To update this information, it is imperative the compilation of all macroseismic data, which is dispersed among several organisms and exist in different formats, being IPMA the responsible for the maintenance of the national seismic network and for the collection of macroseismic information.

The out-of-date and incompleteness of the macroseismic information mentioned, led the authors of this study to propose recently a new map of maximum macroseismic intensities observed in Portugal mainland using all available information for felt intensities larger than III (Teves-Costa et al, 2019). The database used contains intensity data points (IDP) relative to 1098 earthquakes, occurring between 1344 and 2015, for a total of 5444 points (Figure 2).

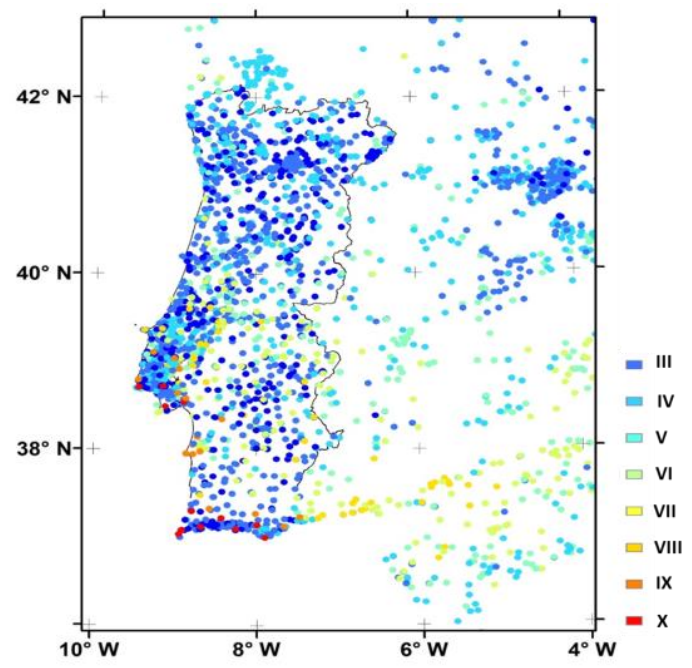

Figure 2. Geographical distribution of the collected IDP

Geographic Information System (GIS) technology was used to compile and process all seismic data. After georeferencing all IDPs in GIS, it was found some spatial heterogeneities, related with the spatial density of IDP's. Many localities, where the intensity is not known, were identified. This fact does not mean that the earthquake was not felt, but only that the intensity was not reported. For this reason, a methodology based on kriging interpolation was developed by the authors in order to produce a continuous surface of maximum intensity throughout the national territory (Figure 3a). This method was considered adequate by several authors who performed similar work with satisfactory results (Schenková et al., 2007, De Rubeis et al., 2005, 2016, Rey et al., 2018). It should be noted that, if the values of maximum intensity observed were higher than the values estimated by interpolation at each site, the first ones were those adopted for the final map. Additionally, data set was also separated on near and far earthquakes which allowed the production of two other maps where the influence of near and far seismic sources was considered separately (Figure $3 \mathrm{~b}$ and $3 c)$. The final macroseismic intensity maps were then, spatially, integrated in a GIS with the administrative areas, in order to attribute a maximum value of intensity to each parish and municipality of the continent. These documents constitute a fundamental tool in the management of seismic risk in each location.

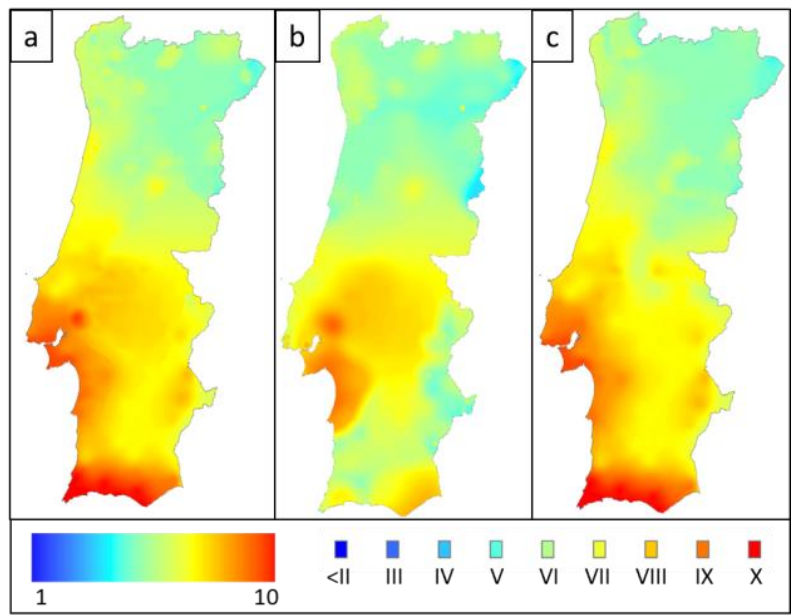

Figure 3. Maximum Intensity Maps (MIM) associated to a) ALL earthquakes; b) NEAR earthquakes and c) FAR earthquakes.

Despite the importance of these maps in seismic risk management, they represent only the maximum intensities estimated or observed in the mainland territory. The aim of this study is to apply spatial pattern analysis techniques to seismic intensities in Portugal mainland to detect clusters and explore global and local spatial patterns related to earthquakes occurred between 1344 and 2015. While in the study of the maximum intensities the authors defined a methodology to spatialize maximum intensity values, which results were concretized in a set of maps representing the worst-case scenarios on the basis of the historical seismicity of almost 700 years, the present study explores the same data catalogue but aims to spatialize seismic intensities which reveals lower intensities values but higher frequencies of occurrence. Kernel density estimation (KDE), Local Moran's I and Getis-Ord general G*, are the spatial analysis techniques chosen for this study.

This work is still presented as a preliminary study since it needs a more detailed analysis and discussion with the introduction of geophysical context to the results and detailed interpretation, not yet performed, on the analysis of spatial correlation with other geographic variables.

\section{SPATIAL STATISTICAL ANALYSIS}

\subsection{Spatial Statistics}

Identifying spatially distributed point patterns plays an important role in many scientific areas including pattern recognition, geomarketing, social sciences, epidemiology, image processing, among others (Diggle, 1993, Gatrell, 1990). Geographic observations are often unevenly distributed in space, not always explicitly presenting trends in their distribution (for clustering or dispersion), or spatial interdependence among observations in a given neighbourhood. Methods that reveal other insights about the data are needed. Spatial statistics is able to suggest important considerations for geophysical researches, making explicit some geographical patterns embedded in available data, creating new views of the data (for example, showing variations in density of points), 
exploring the structure of the data in order to estimate the appropriate models that explain the origin of the observed event distribution. It provides a variety of tools for visualization, exploration and modelling geographic data. When applied to geological and geophysical data, spatial statistics allow us to explore relationships between observations and other geological features represented by points, lines or areas. Spatial statistics takes also advantage of GIS technology to numerically test the spatial randomness hypothesis (Complete Spatial Randomness, CSR) (Diggle, 1983, Cressie, 1991). The CSR model uses some assumptions and several methods to ascertain whether there is a tendency for events to exhibit a random, systematic or clustered pattern. Under CSR, events are independent and the number of events in any specified area of fixed size is Poisson distributed (Gatrell, 1990). The point pattern extracted from any dataset describing locations of observed points, generally in twodimensional space, is compared to those that are derived from a CSR process. If the data exhibits complete spatial randomness, this implies that there is no underlying structure in the data and therefore little to be gained from further analysis.

\subsection{Kernel Density Estimator}

Kernel density estimation (KDE) is used widely to identify hotspot maps which reveal the areas exhibiting a high occurrence of point incidents. In statistics, KDE is a nonparametric way to estimate the probability density function of a random variable. KDE has been popular in many areas related to earthquake engineering (e.g. Woo, 1996, Stock and Smith, 2002, Chen et al., 1998, Karaburun and Demirci, 2016). In this study, KDE is based on the quartic kernel function described in Silverman (1986). Being $i=1, \ldots, \mathrm{n}$ the input points (only include points in the sum if they are within the radius distance of the (x, y) location), the kernel function is given by (1):

$$
\hat{f}(x, y)=\frac{1}{n h} \sum K\left(\frac{d_{i}}{h}\right)
$$

where $\hat{f}$ is the density value at location $(\mathrm{x}, \mathrm{y})$

$n$ is the number of events

$d_{i}$ is the distance between point $i$ and the $(x, y)$ location

$h$ is the smoothing parameter or bandwidth

$\mathrm{K}$ is a density function given by (2):

$$
\begin{gathered}
D(s)=\sum_{i=1}^{n} S_{i}\left(\frac{3}{\pi \tau^{2}}\right)\left[1-\left(\frac{h_{i}^{2}}{r^{2}}\right)\right]^{2}, h_{i} \leq r \\
D(s)=0, h_{i}>r
\end{gathered}
$$

where $\quad r$ is radius of the neighbourhood circle $d_{i}$ is distance between feature $i$ and point location $h_{i}$ is the smoothing parameter or bandwidth $S_{i}$ is the population field value of feature $i$ (equals 1 or a quantity)

The calculated density is then multiplied by the number of points, or the sum of the population field if one is provided. This correction makes the spatial integral equal to the number of points (or sum or population field) rather than always being equal to 1 (ESRI, 2019). In this work the radius was calculated based on Silverman's Rule-of-thumb bandwidth estimation formula but it has been adapted for two dimensions and the population field was set to 1 .

\subsection{Spatial Autocorrelation}

While, first-order properties of a point pattern describe variations in the expected point pattern due to changes in the substantive properties of the local environment, the secondorder effects describe the interactive effects of events explaining on how the events interact. Spatial autocorrelation helps understand the degree to which one object is similar to other nearby objects.

\subsubsection{Moran's I Index}

The Moran's I index is one of most used spatial autocorrelation indicators in the domain of geographic data (Moran, 1950). Global Moran's I calculates an index value that ranges between -1 and +1 using the location of observed data and attribute values to reveal spatial clustering. It assesses overall clustering of data and does not inform on the type, extent and location of spatial clusters and outliers. Local Moran's $I$ is applied to detect the locations of clustered areas with extreme values and allows assessing the significance of local spatial patterns (Anselin, 1995). The local Moran statistic of each observation $I$ is defined as follows:

$$
I_{i}=\frac{Z_{i}}{m_{2}} \sum_{j} W_{i j} Z_{j}
$$

where, $Z_{i}$ is the deviation of the variable of interest with respect to the mean

$W_{i j}$ is the matrix of weights that in some cases is equivalent to a binary matrix with ones in position $i, j$ whenever observation $i$ is a neighbour of observation $j$, and zero otherwise;

$m_{2}=\frac{\sum_{i} Z_{i}^{2}}{N}$

being $N$ is number of analysis units in the map.

A small $p$-value (such as $p<0.05$ ) indicates that location $i$ is associated with relatively high values in surrounding locations. A large $p$-value (such as $p>0.95$ ) indicates that location $i$ is associated with relatively low values in surrounding locations. The local Moran's $I$ and its standardized $z$-score provides an assessment of the similarity of each observation with that of its surroundings (Anselin, 1995, Cliff and Ord, 1981). For each location, a map is produced and classified into five types of association:

- Locations with high values of the phenomenon and a high level of similarity with their neighbours (high-high), defined as "hot spots";

- Locations with low values of the phenomenon and a low level of similarity with their neighbours (low-low), defined as "cold spots";

- Locations with high values of the phenomenon and a low level of similarity with their neighbours (high-low), defined as "potential spatial outliers";

- Locations with low values of the phenomenon and a high level of similarity with their neighbours (low-high), defined as "potential spatial outliers";

- Locations devoid of significant autocorrelations.

\subsubsection{Getis-Ord Gi*}

The Getis-Ord $\left(G_{i}^{*}\right)$ index is also a local indicator of spatial autocorrelation, which identifies hot spots, such as areas of very high or very low values that occur near one another (Getis and 
Ord, 1992). The high/low clustering is an inferential statistic, which means that the results of the analysis are interpreted within the context of the null hypothesis, which states that there is no spatial clustering of feature values. The resultant $z$-scores and $p$-values tell where features with either high or low values cluster spatially. When the $p$-value is small and statistically significant, the null hypothesis can be rejected, otherwise the spatial attribute under analysis presents a completely random distribution in space. Positive and larger z-scores indicate more intense clustering of high values (hot spot) and negative and smaller $z$-score means more intense clustering of low values (cold spot). A z-score near zero indicates no apparent spatial clustering. The results of this index differ from the results of the Local Moran's $I$. While $G i^{*}$ statistic can be used to indicate whether high or low values are concentrated over the study area, Moran's $I$ only indicates that similar values occur together. It does not indicate whether any cluster is composed of high or low values. The $G i^{*}$ statistic is defined as follows (Anselin, 2019):

$$
G_{i}^{*}=\frac{\sum_{j} W_{i j} x_{j}}{\sum_{j} x_{j}}
$$

where $\quad W_{i j}$ is the matrix of weights, $x_{j}$ is the attribute value for feature $j$

\section{RESULTS AND DISCUSSION}

\subsection{Spatial distribution pattern of seismic intensities}

In previous studies, the seismic intensities, derived from far and near seismic sources, were analysed separately by the authors (Teves-Costa et al, 2019). In the present analysis that separation was not yet done, being the main goal focused on the understanding of the spatial pattern distribution of the intensities over the mainland territory. Thus, all IDP's of the initial database contribute simultaneously to the results here presented.

Figure 4 shows the hot spot patterns of the intensities reported using KDE, accordingly it's the level of intensity. The density estimations were normalized, in a scale between 0 and 1 , for better comparison. Although the entire continental territory is affected by seismic activity, originated by near or far seismic sources, the lower intensities $(<\mathrm{VI})$ are mostly located in the northern region of the country. On the other hand, the highest intensities always occur in the central coastal region and in the south of the country (Algarve) being this region, the most affected by earthquakes of high magnitude due to the proximity of the Nabian and Eurasian plate boundary. In addition, it is also verified that the region with the highest population density in the country (Lisbon region) always shows high seismicity with low, high and very high intensities (Figure 5).

This KDE analysis shows also an additional interpretation to the map of maximum intensities produced by Teves-Costa et al. (2019), which reveals the maximum values felt in each location, even if it occurred only once. This fact is particularly well illustrated when comparing images of Figure 2 with the KDE maps, for the central region of the country. The maximum intensities (ranging from VI to XI) in Figure 2 are dominated by the intensity of the earthquake of $\mathrm{Mw} 6.0$ occurred in 1909.04.23 and located NE of Lisbon, near Benavente (TevesCosta et al, 2017).

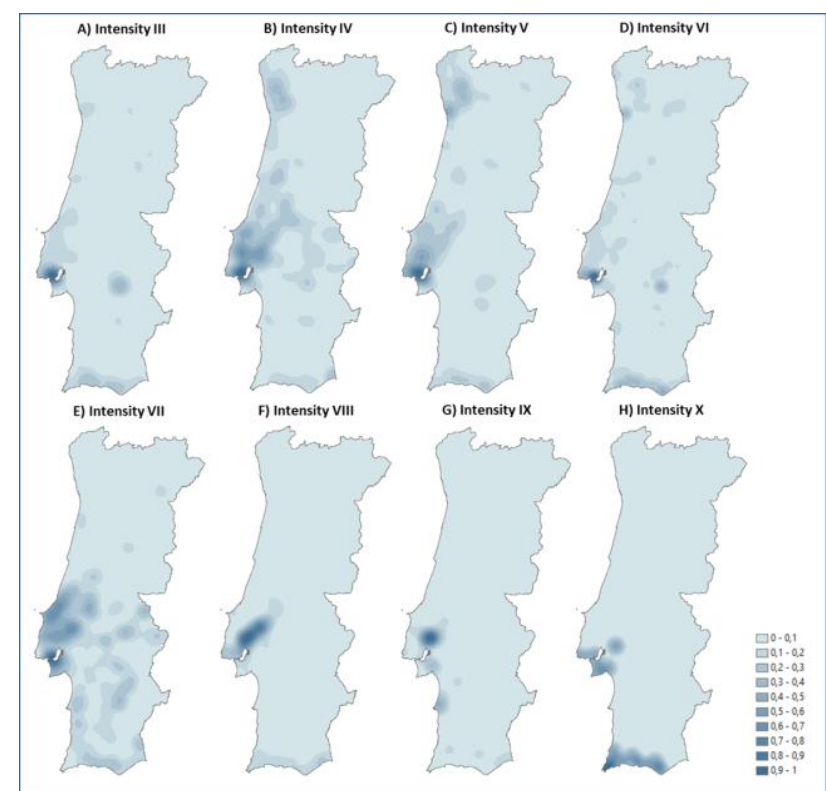

Figure 4. Kernel density estimation (KDE) applied to the earthquake events for all IDP's

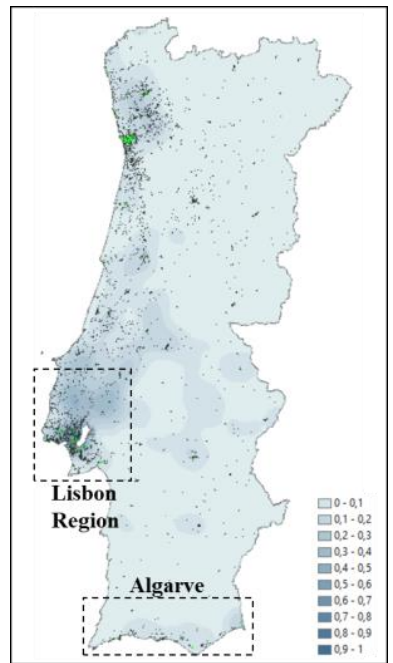

Figure 5. KDE for intensity VI overlaid with the location of the urban areas in black polygons (continuous urban fabric) and green (discontinuous urban fabric). Lisbon region and Algarve

(in the dashed box) are the regions where higher seismic intensities occur

Observing the density of IDP's in this region, is verified that is not so extensive or so obvious the spatial dispersion of such high intensities. However, in risk management, whatever it is, the most likely scenario should not be overlooked relatively to the worst-case scenario. Local decision-makers must have several tools in order to take appropriate measures that best suit each region for risk management, and as such, all tools should be considered and should complement each other.

\subsection{Local spatial autocorrelation}

The local statistics applied in this study identify spatial variation in the relationships between variables, in particular for identifying the presence of clusters or hot spot and for testing 
the assumptions of heterogeneity. The two local statistics considered in this paper are: Local Moran's $I$ and $G i^{*}$ statistic. Figure 6A shows the location of IDP's with significant Anselin local Moran's I statistics, which were applied to identify significant clusters or spatial outliers using the degree of spatial autocorrelation of seismic intensity values. The red dots show observations with high intensities which are surrounded by observations with high intensities. On the other side, the blue dots, show low intensities surrounded by other low intensity observations. The light red and light blue dots indicate the presence of outliers (i.e. high intensities surrounded by low intensities and vice versa) and grey dots are not significant observations. There are clearly spatial clusters in the mainland territory of Portugal. Intensities have a SW-NE distribution with higher intensity events in Lisbon and Tagus valley regions, which is covered by one high-high cluster. Clusters of low intensities are very well demarcated in the NE region of Portugal, and in the interior south. Corroborating the previous analyses with $\mathrm{KDE}$, the Algarve region presents an evident cluster of low intensities, which does not mean that is not affected sporadically by earthquakes of high magnitudes.

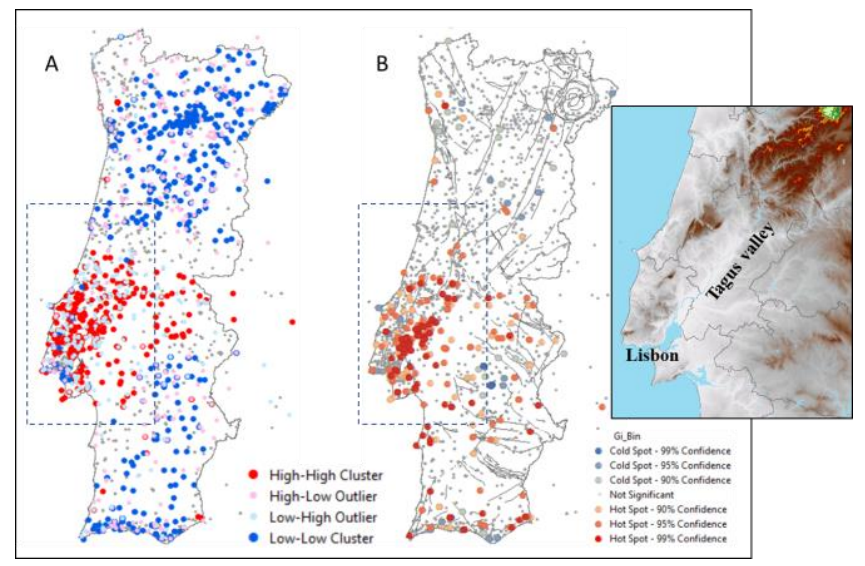

Figure 6. A. Cluster analysis using the Anselin local Moran's I and B. hot spot analysis using Getis-Ord $G i$ * with a representation of the main geological faults. On the right: a Digital Elevation Model of Lisbon and Tagus valley regions, representing the same area in dashed boxes

The red and the blue dots in Figure 6B indicate hot and cold spots, respectively. The Getis-Ord $G i^{*}$ analysis suggests that there are statistically significant hot spots in Tagus valley and a small cluster in the south. Significant cold spots are scarce or non-existent throughout the territory, being the majority of the observations classified as non-significant, which reveals the heterogeneity of the seismic intensities all over the country, where Lisbon and Tagus valley regions are considered exceptions.

One of the most interesting conclusions of this analysis is the strong spatial correlation of the clusters identified by the Local Moran's $I$ and $G i *$ statistic, with other physical geographic variables, in particular with the local geology and the most important geological lineaments. Figure 7 shows a zoom of two different regions, one with a high-high cluster (Lisbon and Tagus valley) and the other with a low-low cluster (Algarve). The correlation between the boundaries of these clusters and the boundaries of specific geological structures is obvious and deserves further analysis in greater detail.

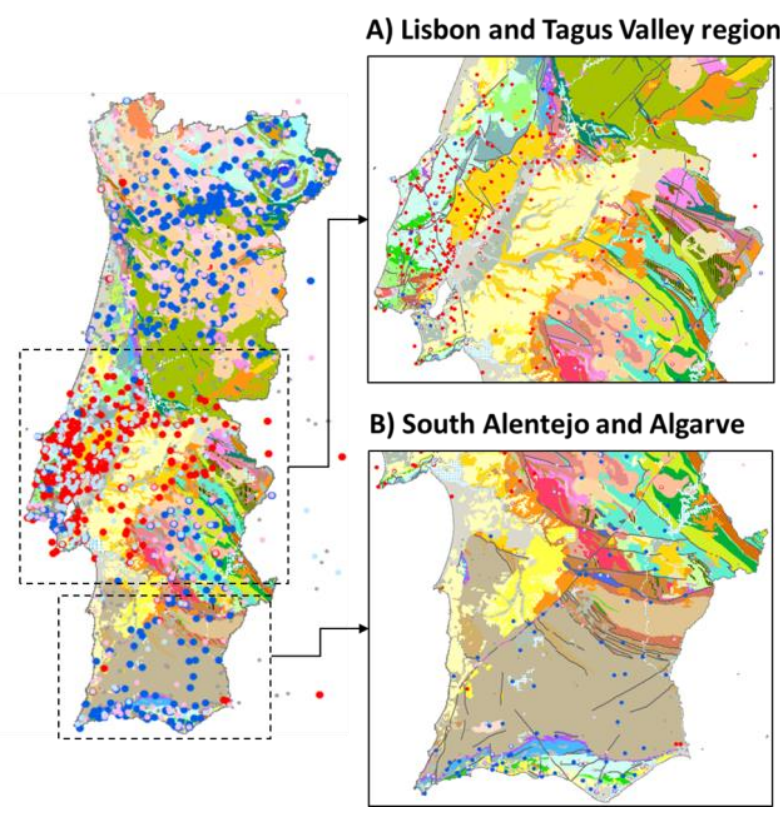

Figure 7. Cluster analysis of Anselin local Moran's I with representation of geological structures (Geological map of Portugal, LNEG)

On the other hand, the analysis of $G i^{*}$ statistic reveals preferential directions of the main hot spots consistent with some of the most relevant geological lineaments (Figure 8).
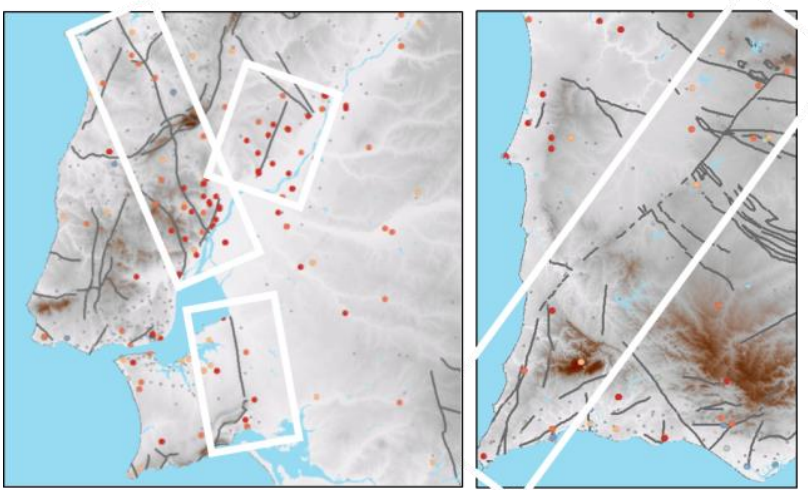

Figure 8. Hot spot analysis of Getis-Ord $G i *$ with a representation of the main geological faults. Left: Lisbon and

Tagus valley regions. Right: SW Portugal. White boxes highlight locations where spatial correlation between hot spots and geological lineaments orientations is strong.

Based on the results of applying Anselin local Moran's $I$ and Getis-Ord $G i^{*}$ statistics, earthquakes with moderate and high intensities are concentrated in areas located in Lisbon and Tagus valley, which is widely known as a seismically active region (Vilanova and Fonseca, 2004). Getis-Ord $\mathrm{Gi}^{*}$ and local Moran's $I$ provide different and complementary information about the spatial clusters, being Getis-Ord $G i^{*}$ an indicator of greater certainty of the location of the hot spots, i.e. the zones of higher seismic risk.

\section{CONCLUSIONS}

The aims of this preliminary study are the exploitation of some spatial statistic techniques and GIS technology to reveal local 
evidences related to seismic activity in the Portugal mainland, which are not commonly analysed or evidenced by the classical interpretive methods. All techniques used show the presence of clusters of intensity values in specific regions of the country, with a greater predominance in Lisbon and Algarve regions regarding the higher intensities. The northern region is less affected as evidencing clusters of low values of intensity. These preliminary results prove that spatial statistics analysis can constitute a valuable method to show complex and unrevealed spatial phenomena distributions and associations. This study needs a greater analytical investment regarding to the correlation of these results with the reality of the terrain, in particular with the geology, tectonics and other processes related with the Earth dynamics. This and further studies that follow this line of research, may constitute a key contribution to the updating of the existing seismic risk maps.

\section{ACKNOWLEDGEMENTS}

Publication supported by FCT- project UID/GEO/50019/2019 Instituto Dom Luiz.

\section{REFERENCES}

Anselin, L., 1981: Spatial Econometrics: Methods and Models; Kluwer Academic: Dordrecht, The Netherlands, 1988.

Anselin, L., 1995: Local Indicators of Spatial AssociationLISA. Geogr. Anal, 27, 93-115.

Anselin, L., 2019: Local Spatial Autocorrelation, Univariate Local Statistics. GeoDa Resources on-line:

https://geodacenter.github.io/workbook/6a_local_auto/lab6a.ht ml\#getis-ord-statistics. Accessed: 5 Maio 2019.

Campos A, Sousa ML, Carvalho A., 2008: Seismic zoning for Portuguese national annex of Eurocode 8. Proc. 14th World Conf Earthq Eng, Beijing, China.

Chen, Y., Liu, J., Chen, L., Chen, Q., Chan, S., 1998: Global seismic hazard assessment based on area source model and seismicity data. Natural Hazards, 17, 251-267.

Cliff, A. and Ord, J., 1981: Spatial Processes: Models and Applications; Pion Limited: London, UK, 266 p.

Cressie, N. A. C., 1991: Statistics for spatial data John Wiley, Chichester.

De Rubeis, V., Tosi, P., Gasparini. C., Solipaca, A., 2005: Application of kriging technique to seismic intensity data. Bull Seism Soc Am 95(2): 540-548.

De Rubeis, V., Sbarra, P., Tosi, P., 2016: Regional macroseismic field and intensity residuals of the August 24, 2016, Mw=6.0 central Italy earthquake. Annals of Geophysics, Vol. 59, DOI: https://doi.org/10.4401/ag-7217.

Diggle, P.J., 1983: Statistical Analysis of Spatial Point Patterns. Mathematics in Biology, Vol. 2. Academic-Press, London New York, pp 148.

ESRI, 2019: ArcGIS Pro, Resources online: https://pro.arcgis.com/en/pro-app/tool-reference/spatialanalyst/how-kernel-density-works.htm, Environmental Systems Research Institute, Redlands, CA. Accessed: 20 June 2019)
Gatrell, A.C., 1990: On modelling spatial point patterns in epidemiology: cancer of the larynx in Lancashire. Research Report No. 9 North West Regional Research Laboratory, Lancaster University

Getis, A., and Ord., J. K., 1992: The Analysis of Spatial Association by Use of Distance Statistics. Geographical Analysis 24, no. 3. 1992.

IPQ, 2010: Eurocódigo 8 - Projeto de Estruturas para resistência aos sismos. Parte 1: Regras gerais, ações sísmicas e regras para edifícios. NP EN 1998-1, Inst. Port. da Qual. [in Portuguese]

Karaburun, A. and Demirci, A., 2016: Spatio-temporal cluster analysis of the earthquake epicenters in Turkey and its surrounding area between 1900 and 2014. International Journal of Research In Earth \& Environmental Sciences, Feb. 2016. Vol. 4. No.1.

Moran, P.A.P., 1950: Notes on Continuous Stochastic Phenomena. Biometrika. 37 (1): 17-23. doi:10.2307/2332142. [S.1.], nov. 2016. ISSN 2037-416X. doi:http://dx.doi.org/10.4401/ag-7217.

Rey, J., Beauval, C., Douglas, J., 2018: Do French macroseismic intensity observations agree with expectations from the European Seismic Hazard Model 2013, J Seism 22(3): 589-604.

Schenková, Z., Schenk, V., Kalogeras, I., Pichl, R., Kottnauer, P., Papatsimba, C., Panopoulou, G., 2007: Isoseismal maps drawing by the kriging method. J Seismol 11: 121-129. DOI 10.1007/s10950-006-9023-1.

Silverman, B.W., 1986: Density Estimation for Statistics and Data Analysis. New York: Chapman and Hall, 1986.

Stock, C., and Smith, E.G.C., 2002: Adaptive kernel estimation and continuous probability representation of historical earthquake catalogs. Bulletin of the Seismological Society of America, 92(3), 904-912, 2002.

Teves-Costa, P., Batlló, J., Matias, L., Catita, C., Jiménez, M. García-Fernández, M., 2019: Maximum intensity maps (MIM) for Portugal mainland. Journal of Seismology. 10.1007/s10950019-09814-5.

Teves-Costa, P., Batlló, J., Cabral, J., 2017: The Lower Tagus Valley (Portugal) earthquakes: Lisbon 26 January 1531 and Benavente 23 April 1909. Física de la Tierra 29: 61-84.

Vilanova, S. and Fonseca, J., 2004: A Falha Do Vale Inferior Do Tejo Na Análise Da Perigosidade Sísmica. Sísmica $2004-$ $6^{\circ}$ Congresso Nacional de Sismologia e Engenharia Sísmica, Guimarães, 14, 15 e de Abril 2004.

Woo, G., 1996: Kernel estimation methods for seismic hazard area source modeling. Bulletin of the Seismological Society of America, 86(2), 353-362, 1996. 